Proceedings

\title{
An Oveview and Update on Sarcopenic Obesity
}

\author{
Mauro Lombardo 1,2, Andrea Boaria 1, Giovanni Aulisa 2, Elvira Padua 1,3, Giuseppe Annino 1,3, Antonio Pratesi ${ }^{4}$, \\ Massimiliano Caprio 1, Ferdinando Iellamo 2,3, Alfonso Bellia 1,2
}

Publisher's Note: MDPI stays neutral with regard to jurisdictional claims in published maps and institutional affiliations.

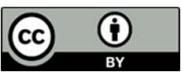

Copyright: ( 2020 by the authors. Submitted for possible open access publication under the terms and conditions of the Creative Commons Attribution (CC BY) license (http://creativecommons.org/licenses /by/4.0/).

\author{
${ }^{1}$ Department of Human Sciences and Promotion of the Quality of Life, San Raffaele Open University, Rome, \\ Italy. \\ ${ }^{2}$ Department of Systems Medicine, University of Rome “Tor Vergata," Rome, Italy. \\ ${ }^{3}$ School of Human Movement Science, University of Rome "Tor Vergata," Rome, Italy. \\ ${ }^{4}$ Local Health Authority - ULSS n. 2 - Treviso, Italy \\ * Correspondence: mauro.lombardo@uniroma5.it
}

\begin{abstract}
Sarcopenic obesity (OS) is a multifactorial condition characterized by the simultaneous presences of sarcopenia and obesity. It represents a natural phenomenon related to aging that can significantly increase the risk of osteoporosis, frailty, mobility, disability and mortality. It is estimated that approximately $45 \%$ of the elderly American population — about 18 million people — are affected by sarcopenia and that the risk of disability is $1.5-4.6$ fold greater in elderly people suffering from sarcopenia than older people with unaffected muscle mass. The prevalence of OS is increasing in adults over 65 years of age; people with OS present greater health risks than people who are only sarcopenic or obese. Therefore, the study of OS and the search for effective treatment are important due to the constant increase of the elderly population. OS is a relatively new concept in the panorama of age-related diseases. There is currently no single definition of the pathology; however, the evidence suggests a close correlation to the onset of cardiovascular disease and mortality. Although many questions remain unresolved today, it is important to note that OS can be prevented and treated by adopting a healthy lifestyle. The ideal approach is based on the targeted intervention to both slow down the course of sarcopenia and promote a decrease in adipose mass. The beneficial potential of physical activity, expressed through strength and resistance exercises, on OS is widely documented. The benefits obtained from aerobic exercises and strength exercises are shown at the level of muscle mass, muscle strength, muscular capacity and mitochondrial activity. However, the best results were obtained by coupling adequate nutritional treatment with regular physical activity. The adequate intake of proteins high in essential amino acids promotes protein synthesis and muscle health, effectively counteracting the onset of sarcopenia. In case of malnutrition a supplementation with BCAA-based supplements, leucine, vitamin D and $\beta$-HMB might help to improve and reduce the effects of sarcopenia. The deficiency of muscle mass in obese elderly subjects will be one of the health challenges of the future to reduce the risk of chronic diseases.
\end{abstract}

